\title{
Implementing The Use of Magic Triangles for Developing Mathematical Reasoning Process in Delivering School Mathematics
}

\author{
$1^{\text {st }}$ Gatot Muhsetyo \\ Universitas Negeri Malang \\ Malang, Indonesia \\ gatot.muhsetyo.fmipa@um.ac.id
}

\begin{abstract}
The pattern of perimeter magic triangles has not yet implemented by teachers for delivering mathematical reasoning. The purpose of this implementation is to describe learning practice for students of Elementary Education Master Program with a mathematics concentration. It is also as a part in mastering mathematics and developing mathematics in elementary school. The study was performed by using qualitative study with 17 students, 13 of them come from outside East Java. The implementation is done in two sessions. The first session is used to introduce and then explore number patterns, and the second session is used to discuss reasoning processes in determining number arrangements in perimeter magic triangles. The second session problem can be classified as a non-routine problem since they never know the problem before, and the way to get the answer needs valid mathematical reasoning. The implementation shows that they gain clarity and in-depth outlook about mathematical reasoning in solving a problem.
\end{abstract}

Keywords-magic triangle, number arrangement, mathematical reasoning

\section{INTRODUCTION}

In solving mathematics problems, someone needs thinking. Although what they think is not seen or cannot be observed, they use particular steps, and these steps are said thinking processes. Stacey writes that in thinking needs substance, not proof [1]. It is also stated by Stacey, that the importance of thinking the substance is reasoning, the way to develop thinking and to make logical action (making sense), such as analyzing, proving, evaluating, citing, justifying, and generalizing [1]. Star states that reasoning is part of understanding when someone is solving a problem, and it can be said that reasoning is the capacity (a) in applying concepts, (b) in relating facts and concepts, and (c) in making decisions [5]. Stacey states that basic mathematical capability includes doing communication, implementing representation, designing strategy, using symbols, and reasoning argumentation [2].

It is predicted that many teachers tend to hesitate in delivering healthy and qualified mathematics learning. Stacey says that this kind of low motivation is named as Shallow Teaching Syndrome (STS) [2]. Stacey also identifying the phenomena of STS are (1) repetition of previous problems, (2) low procedural complexity, and (3) the absence of mathematical reasoning [2]. Repetitions are known by identifying teacher actions in which the teacher using the textbook, the materials, and the problems, without preliminary inspection. Inspection needs to be accomplished in the three conditions. Firstly, the available materials and the problems are only routine works and exercises. The second, the materials and the problems can be modified to be non-routine texts and problems, such that students can develop their competence in reasoning and argumentation. Lastly, the materials and problems can push students to be more challenged and motivated to find the answers. Using inspection, teachers have a purpose for making students learn mathematics, and bringing them to think profoundly, not to think partially. Students are also expected to give a reason for any decision and able to change their decision when it is wrong or needs to be completed with different reasons.

Numbers are a unique mathematical object. The uniqueness of numbers can be seen from different point of views. First, numbers can be met everywhere in the everyday social life, such as for counting, for representing the result of measuring (length, area, volume, weight), and business transaction (the number of purchased things, the price of every good, the dimension of..., and the payment). Second, numbers are used for labeling of things, such as numbering houses on the streets, numbering motored vehicle, numbering phones, numbering for people (PIN, Personal Identity Number), and Password for specific needs related to banking and internet activities. Third, numbers are creative human production, like the alphabets which have a particular characteristic that has been explored since the era of Pythagoras about 550 B.C. The focus of exploration is the existence of number patterns that are related to sequences, series, particular arrangement/ pattern, and geometric maps. Number patterns in geometric shapes are frequently said as magic numbers, such as square magic numbers, and triangular magic numbers.

Muhsetyo, says that magic square usually is known and used in delivering school mathematics [6]. Besides, the magic triangle is rarely known and used by mathematics teachers. That is why this article tries to explore the use of numbers pattern in a magic triangle to teach mathematics in school. Magic triangle is especially useful for developing number operation skills, through logical and reasonable processes. The problem of numbers pattern in the magic triangles is positioning numbers $1,2,3, \ldots, \mathrm{n}$ on the triangle sides ( $\mathrm{n}$ is said the dimension of magic triangle) such that the sum of numbers in each side of the triangle are equal (this sum of numbers in one side is said the constant of the magic 
triangle). As an example, observe the numbers arrangement in the 3-dimension magic triangle with its constant is 10 , and in the 4-dimension magic triangle with its constant is 20 (Fig.1).

Several questions that can be used to develop student's reasoning are (a) how the numbers arranged on the triangle sides, (b) how the constant is found, (c) whether the arrangement found is unique (d) whether the strategy for arranging the numbers in 3-dimension magic triangle, is also the strategy for arranging the numbers in 4-dimensions magic triangle?

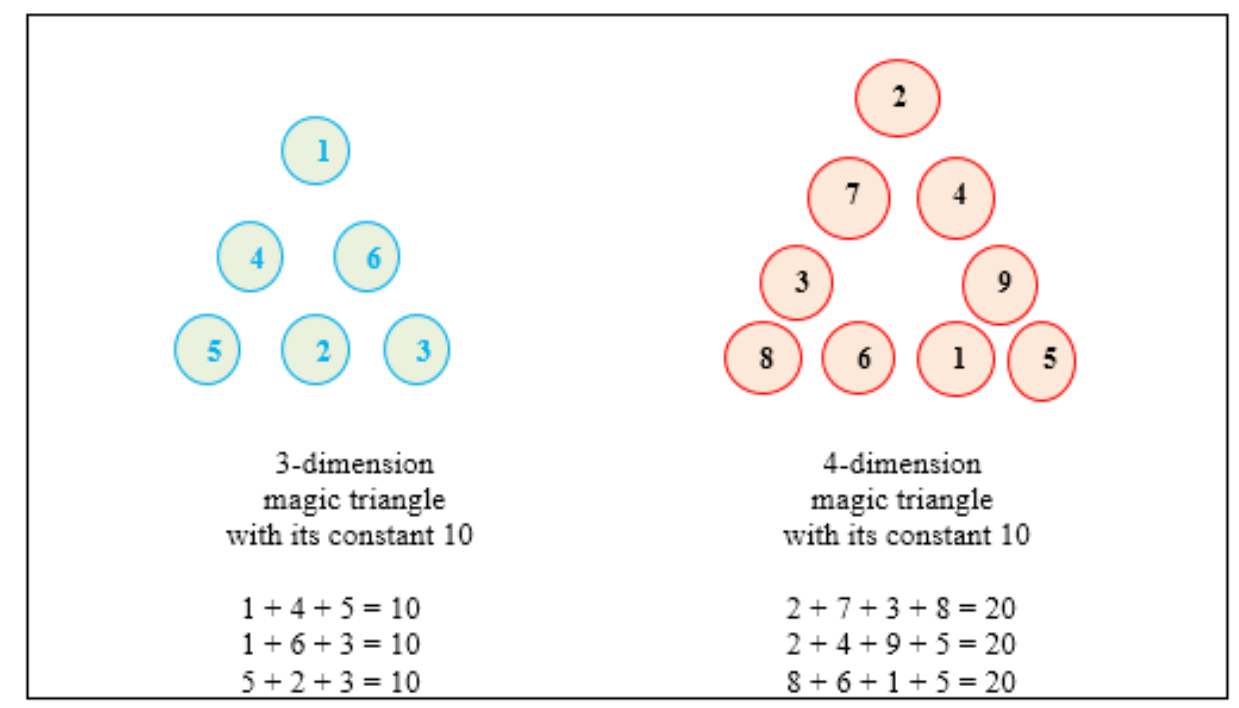

Fig. 1. Example of the numbers arrangement in the 3 -dimension magic triangle with its constant is 10 , and in the 4-dimension magic triangle with its constant is 20 . 


\section{METHODS}

The implementation method of the learning process is a qualitative method in the form of a delivery system. This method can be arranged in three steps, the preparation step, implementation step, and extension step. The preparation is done in the first session (20 minutes), the implementation is done in the first session (70 minutes), and it is continued in the second session (50 minutes). The extension is done in the second session (40 minutes).

\section{A. The Preparation Step}

The Preparation is used to (1) giving students opportunity to remember and understand knowledge about the characteristics of integer and its operations, (2) enriching students about the regular addition problems and open-ended addition problems.

- In 20 minutes, through class discussion, students are asked to recall their knowledge about integers (negative, null, positive, number line), and some principles of number operation (commutative, associative, distributive, vertical addition), as the bases in reasoning and arguing.

- Some focuses can be identified to be related to the effort of finding many ways in solving an addition problem: finding $93+89$ with different ways:

$$
\begin{aligned}
& 93+89=93+(7+82)=(93+7)+82=100+82= \\
& 182 \\
& 93+89=(\mathbf{8 2}+\mathbf{1 1})+\mathbf{8 9}=\mathbf{8 2}+(\mathbf{1 1}+\mathbf{8 9})=\mathbf{8 2}+ \\
& 100=182 \\
& 93+89=(\mathbf{9 2}+\mathbf{1})+\mathbf{8 9}=\mathbf{9 2}+(\mathbf{1}+\mathbf{8 9})=\mathbf{9 2}+\mathbf{9 0}= \\
& (90+2)+90=182 \\
& 93+89=(\mathbf{5 0}+\mathbf{4 3})+(\mathbf{5 0}+\mathbf{3 9})=\mathbf{1 0 0}+(\mathbf{4 0}+\mathbf{3})+ \\
& (30+9)=170+12=182 \\
& 93+89=(\mathbf{1 0 0}-\mathbf{7})+\mathbf{8 9}=\mathbf{1 0 0}+\mathbf{8 9}-\mathbf{7}=\mathbf{1 0 0}+\mathbf{8 2} \\
& =182 \\
& 93=90+3 \\
& 89=\mathbf{8 0}+\mathbf{9} \\
& 93+89=\mathbf{1 7 0}+\mathbf{1 2}=\mathbf{1 8 2}
\end{aligned}
$$

- Modifying regular problems to be open-ended problems:

Regular problem:

$2+5=\ldots$.

$3+7+9=\ldots$

$3+\ldots=11$

$7+\ldots+2=14$

The answer of each problem is single, not more than one solution

Open-ended problem:

$$
\ldots+\ldots=7
$$

$$
\begin{aligned}
& \ldots+\ldots=11, \\
& \ldots+\ldots+\ldots=19 \\
& \ldots+\ldots+\ldots=14
\end{aligned}
$$

The answer to each problem is not single, more than one solutions.

\section{B. The Implementation Step}

Implementation step is used to engage groups of students to solve a problem. The problem is about arranging six numbers $1,2,3,4,5$, and 6 in a 3 dimension magic triangle, without giving them help, product help, nor process help. The available time is 50 minutes.

- The problem:

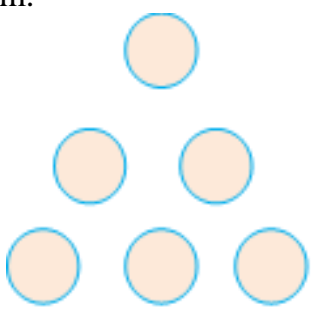

Fig. 2. Magic triangle model

From above model, place numbers "1", "2", "3", "4", "5", and "6" inside the circle such that there is an arrangement in which the sum of three numbers in each side of "triangle" are all the same. Explaining the way you get the right answer. After having one right solution, try to find other solutions. The last 20 minute used to do a class discussion, for sharing the solution of each group.

From the work of the group, it can be understood that they usually use trial and error work, and none of the group has creativity by presenting a different way. Although they are less creative in doing the problem, it can be identified that students develop reasoning. This is because they are familiar about facts, procedures, and concepts when they are doing the trial and error work or a guess and check work. This kind of student responses is suitable with the opinion of Star, that the beginning of reasoning understands about facts, procedures, and concepts [7].

\section{The Extension Step}

Extension step is used to widen the problem of 3dimension magic triangle to 4-dimension magic triangles. In 40 minutes, they are asked to make at least two arrangements of four numbers from 1, 2, 3, $4,5,6,7,8$, and 9, such that the triangle becomes magic triangles. All groups are guided together to use analogy reasoning, reasoning to use the same way to get number arrangement in magic triangles. The analogy reasoning consists of (1) making the sum list of four numbers (without repetition), and (2) 
selecting the appropriate four numbers that satisfied the rule.

\section{RESUlT AND DISCUSSION}

\section{A. The Preparation Step}

When they are asked to find $93+89$, and mention the way to get the sum, in general, their answer is right, but they usually use the same way, the column addition method.

93
89
---
182

F From this fact, it is known that the methods other than the column method are not familiar to the students. It is indicating such that they almost never use, or maybe never know the other way to obtain the sum of numbers, except the column addition method. Although they can completely memorize rules of number addition, they do not understand to use them practically. It can be predicted that in number addition, teachers' knowledge has not changed for years, and it is the same as their teachers guide them in learning number addition. Their knowledge is limited to the technique, and they cannot make their students more creative, and more critical in developing many solutions, and many methods of solution. As a result, the student's creativity are still low, they will be passive, and not skillful when facing complex problems, and to solve the problems in a definite time.

The reasonable capability of students is growing more effective when they accept examples for obtaining the result of $93+89$, and it will improve their reasoning skill while they are presented many methods that are easier and faster when it is compared with column method, even they can use it mentally, without paper and pencil calculation. The advanced way contains (1) using compatible numbers, two or numbers that can be operated easier than usual, and (2) applying the rules of number operation perpetually. After they have experiences in many new methods, in general, the feeling that they get new knowledge that can make their students have many choices in solving mathematics problems.

The students' perspectives become wider and more confident when they are able to solve problems such as

$$
\ldots+\ldots=12 \text { and } \ldots+\ldots+\ldots=10 \text {, }
$$

with the universe is the set of integers. At that time, they begin to realize that in teaching elementary school mathematics, students need to be guided and directed such that they become more creative. Their creativity is build with some problems, such as: $1+11=2+10=3+9=4+8=5+7=\mathbf{1 2}$
$1+2+7=1+3+6=1+4+5=2+1+7=2+3+$ $5=10$

\section{B. The Implementation Step}

In the group discussion, they become enthusiastic to solve the problem faster, but the way to obtain the answer is relatively the same, they usually use trial and error strategy, and it is called random guess test [8]. It is not seen that they use systematic strategy, for instance deciding 1,2, and 3 in the corners, and replacing by $1,2,4$, or $1,2,5$, or $1,2,6$, or $2,3,4$, or $2,3,5$, or $2,3,6$, or etc if it does not work. The inferential strategy does not appear, for example, deciding 3 in a corner, and looking for two other numbers with 3 as the common element when it is placed in two sides. There are two groups trying to find the constant by adding $1+2+3+4+5+6=$ 21 , and then dividing 21 by 3 , and it is obtain 7 . This way is wrong because there is only one triple 1, 2, 4 which the sum is 7 . Although this way is wrong, it needs to be appreciated since they use the same way as finding the sum of numbers in a row, or in a column, when they fill out 3-dimension magic square. It means that they try to use reasoning for solving the problem. Their fail is caused by the existence of 3 as the common number of 2 sides (there is no a common number of two rows or two columns).

Teachers should guide their students to discuss their mathematics ideas [7]. Guidance or scaffolding is needed for the students, and by helping them, it is hoped that their ability of reasoning will develop. The given help is directed to the solution of the problem. According to Pijls, M., and Dekker, R. [7], this kind of help is named product help. The first step is identifying the number of triples, and then it can be formed from 1, 2, 3, 4, 5, and 6. By using a tree diagram, they can obtain all 120 of the triples. If the tree begins with 1 , then they can obtain 20 triples (fig.2).

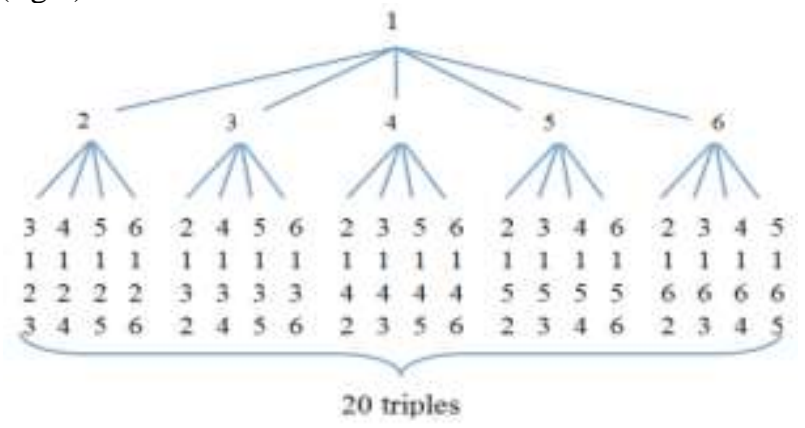

Fig.3. Tree diagram to the help identifying the number of triplets

If 1 is replaced by $2,3,4,5,6$, then the diagram will produce $6 \times 20=120$ triples. 
The next aid is in determining the constants. The constant can be obtained from the list of 120 triples by asking them in order, to reveal the reason why some triples are not satisfying. After finishing this activity, they know the boundaries of constants, its minimum sum, and its maximum sum.

a) the triple 1,2, 3 has a constant 6 , and it can not be used because ... (there is only one triple that its sum is 6 , it is needed three triples).

b) the triple 4, 5, 6 has a constant 15 , and it can not be used because ... (there is only one triple that its sum is 15 , it is needed three triples).

c) from (a) and (b) can be understood that the interval of $k$ is $(6<\mathrm{k}<15)$

d) If $\mathrm{k}=7$, then there is only one triple ... (the triple is only $1,2,4$, its sum is 7 , it is needed three triples).

e) If $\mathrm{k}=14$, then there is only one triple ... (the triple is only $3,5,6$, its sum is 14 , it is needed three triples).

f) from (d) and (e) can be understood that the interval of $\mathrm{k}$ is $(7<\mathrm{k}<14)$

g) If $\mathrm{k}=8$, then there are only two triples... (the triples are only $1,3,4$, and $1,2,5$, it is needed three triples). h) If $\mathrm{k}=13$, then there are only two triples... (the triples are only $3,4,6$, and 2, 5, 6, it is needed three triples).

i) from $(\mathrm{g})$ and $(\mathrm{h})$ can be understood that the interval of $\mathrm{k}$ is $(8<\mathrm{k}<13)$

Its minimum $\mathrm{k}$ is 9 , and its maximum $\mathrm{k}$ is 12 .

From the product help (a) to (i), they know that the possibility of $\mathrm{k}$ is $\mathrm{k}=9,10,11$, and 12 . In succession, every group is asked to solve the problem, from $\mathrm{k}=9$ to $\mathrm{k}=12$. When $\mathrm{k}=9$, every group is asked to identify all triples that each of its sums is 9 , and it will be obtained $[(1,3,5),(1,2,6),(2,3,4)]$. From these three triples, they are asked to reason, how one places the numbers to form a magic triangle. There is no answer from the students. It means that they need help. The product help is given by asking them to notice the common element of the available tree triples and the meaning of the common elements. The common element of : $(1,3,5)$ and $(1,2,6)$ is $1,(1,2$, $6)$ and $(2,3,4)$ is $2,(1,3,5)$ and $(2,3,4)$ is 3 . So, the common elements of two triples are 1,2 , and 3 . The meaning of the common elements 1,2 , and 3 is their position is on the corners of the triangle. At last, each group is asked to draw a magic triangle with $\mathrm{k}=9$

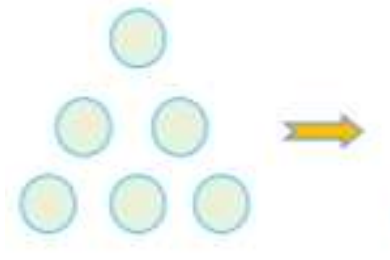

Fig. 4. Triplets number placement in the magic triangle model

Without asking, every group actively continue to arrange numbers to obtain magic triangles which have constant $\mathrm{k}=10,11$, and 12 . They use analogous thinking, beginning with identifying the suitable triples, listing the numbers, finding the common numbers among triples, completing activity by
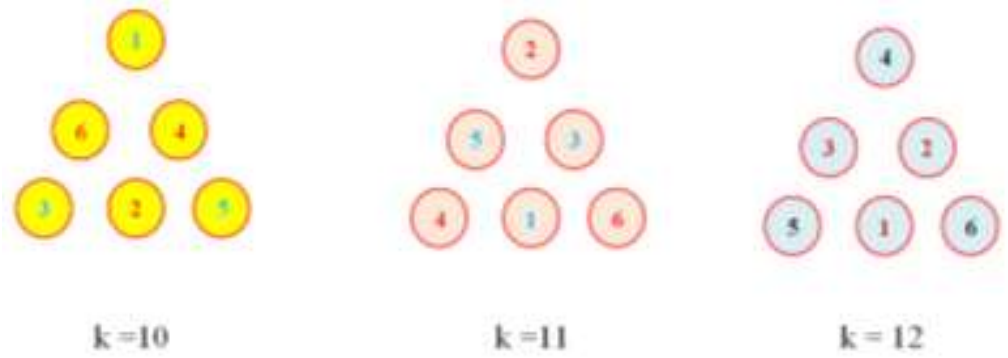

Fig. 4. Magic triangle pattern for constant $\mathrm{k}=10,11,12$ C. The Extension Step

In the extension step, the topic about 3-dimension magic triangles is extended to be 4-dimension magic triangles. The instruction before is talking about

triples, and the next instruction will talk about quadruples. The numbers of each quadruple are taken from $1,2,3,4,5,6,7,8,9$. Using analogous reasoning, they are asked to obtain at least two

placing common numbers in the corner, and at last placed the other numbers not in the corners. They look like more skillful, more active, and faster in finding the answers. At the end of their discussion, every group can complete the other three solutions, as shown below.

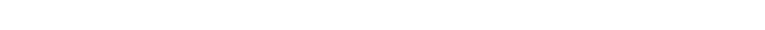


number arrangements of 4-dimension magic triangles. From the beginning, they realize that a random guess and testway is not effective to be used since needs more time. The possible constants $\mathrm{k}$ in

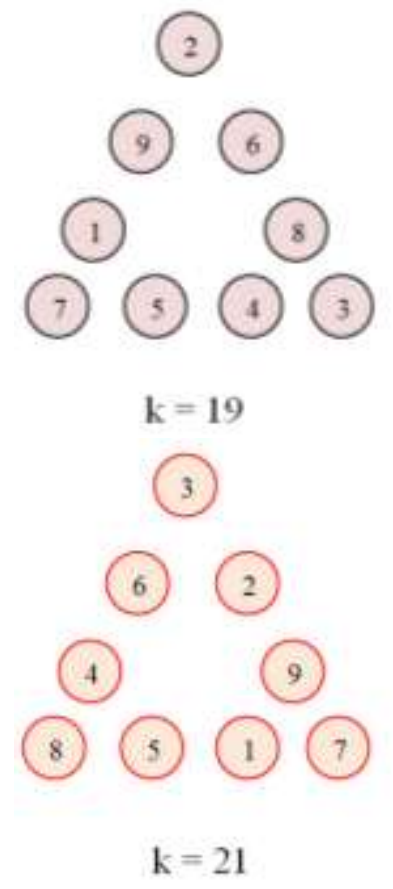

\section{CONCLUSION}

The important part of mathematical works is reasoning. Reasoning activity can be identified by the increase of thinking capability and logical action, such as analyzing data and information, giving reasonable arguments (making sense), evaluating possible ways to be chosen, finding good references as the bases of the arguments, and generalizing form of trends from cases. It is a reality that the implementation use of number arrangement in the magic triangle can be a tool to develop mathematical reasoning. After having the learning experience about this implementation, in the future, after completing their study, they will be a dynamic agent of changes, especially in delivering school mathematics. They also have experience that the use of magic triangles will make students (a) have fun, (b) not afraid to mathematics, (c) to have reasoning habit, (d) skillful in doing number operation, and (e) to have good perspectives about open-ended problems. They feel that they get fresh air in providing them knowledge, skill, and attitude, to be more implemented by other teachers in delivering school mathematics.

\section{REFERENCES}

[1] K. Stacey, "Mathematics Teaching, and Learning To Reach Beyond The Basics," Research Conference the interval $10<\mathrm{k}<30$. Some solutions with $\mathrm{k}=19$, 20, 21, 23, (Muhsetyo, G., 198) can be seen as follows:

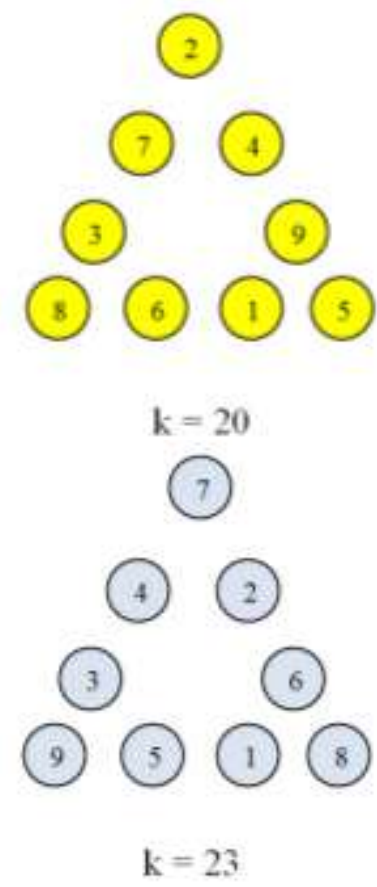

Paper, Australia: Australia Council for Educational Research, 2010.

[2] M. Pijls, and R. Dekker, Students discussing their mathematical ideas: the role of the teacher. Mathematics Education Research Journal vol.23 (2011)23:379-396.

[3] K. Stacey, PISA 2012: Framework and Items. Seoul: The International Assesment of Mathematical Literacy, 2012.

[4] G. Muhsetyo, Menghayati Kekayaan dan Keindahan Matematika, Malang:Universitas Negeri Malang, 2014.

[5] J. Star, "What is mathematical understanding? How can we measure it? Theoretical and empirical," Seminar Presentation. Ankara, Turkey: Middle East Technical University, 2007.

[6] G.L. Musser, W.F. Burger, and B.E. Peterson, Mathematics For Elementary Teachers A Contemporary Approac, Hoboken: John Wiley \& Sons, 2004. 